\title{
JULIA SETS AND DIFFERENTIAL EQUATIONS
}

\author{
HAROLD E. BENZINGER
}

(Communicated by Kenneth R. Meyer)

\begin{abstract}
A one-parameter family of Julia sets is shown to converge, in a probabilistic sense, to certain trajectories of a differential equation. The Julia sets arise from Euler's method for the differential equation. This provides information on the location of the Julia sets and the dynamics on them.
\end{abstract}

\section{INTRODUCTION}

Let $R$ denote a rational function. We study the continuous dynamical system

$$
\dot{z}=R(z) \quad(\dot{z}=d z / d t)
$$

and the discrete dynamical system

$$
z_{n+1}=z_{n}+h R\left(z_{n}\right),
$$

where $t, h$ are real parameters, $h>0$. Let

$$
N(z, h):=z+h R(z),
$$

so that (1.2) takes the form

$$
z_{n+1}=N\left(z_{n}, h\right) .
$$

Clearly, $N(\cdot, h)$ is also a rational function. Both dynamical systems are to be considered on the Riemann sphere $\widehat{\mathbf{C}}$.

In this paper we show that for $h$ small, the Julia set $J(h)$ of $N(\cdot, h)$, and the dynamics on the Julia set, are related to the phase plane portrait of the differential equation (1.1).

The discrete dynamical system is Euler's method for the approximation of solutions to (1.1) by polygonal paths. Thus, if $z_{0}$ is given and $\varphi\left(t, z_{0}\right)$ denotes a solution to (1.1) such that $\varphi\left(0, z_{0}\right)=z_{0}$, then the polygonal path obtained by connecting successive points $z_{k-1}, z_{k}$ by straight line segments, $0 \leq k \leq K$, is expected to be close to $\varphi\left(t, z_{0}\right)$ for $0 \leq t \leq K h$, with the approximation improving for smaller $h$, keeping $K h$ fixed. See [9, Chapter 1] for a survey of Euler's method.

Received by the editors August 8, 1989.

1980 Mathematics Subject Classification (1985 Revision). Primary 34C35, 34A20, 58F13, 65L05. 
There is another interpretation of these dynamical systems. In [2, Theorem 2.3 ] it was shown that for any rational function $R$, there exists a function $f$, unique up to a nonzero multiplicative constant, such that

$$
R(z)=\frac{f(z)}{f^{\prime}(z)} .
$$

Then (1.1) and (1.2) take the respective forms

$$
\begin{gathered}
\dot{z}=-\frac{f(z)}{f^{\prime}(z)}, \\
z_{n+1}=z_{n}-h \frac{f(z)}{f^{\prime}(z)} .
\end{gathered}
$$

The function $f$ is analytic in the finite plane except for at most finitely many poles, essential singularities, and branch points. In the form (1.6), the continuous dynamical system is the continuous Newton method for $f$. See [2] for results and further references. In forward time, solution curves $\varphi\left(t, z_{0}\right)$ travel from places where $|f|$ is large to places where it is small. This can be seen most easily from the implicit solution $f\left(\varphi\left(t, z_{0}\right)\right)=f\left(z_{0}\right) e^{-t}$. Since $f$ is in general multivalued, the preceding statement must be interpreted carefully. See [2].

In the form (1.7), with $h=1$, the discrete dynamical system is the familiar Newton method for $f$, and for $h \neq 1$ it is the modified or relaxed Newton method. See [11]. See [5] for results on the invariant measure on Julia sets, and see [4] for a general survey of rational dynamics. In [2] and in this paper, the differential equation (1.1) is considered without desingularization. See [10] for desingularized equations arising from meromorphic functions.

\section{Preliminaries}

We collect here some results about (1.6) and (1.7). A point $\gamma$ in the finite plane is a critical point of (1.6) if $f(\gamma) \neq 0, f^{\prime}(\gamma)=0$. The point at $\infty$ is a critical point of $(1.6)$ if 0 is a critical point of

$$
\dot{z}=-z^{2} R(1 / z),
$$

the system obtained from the inversion $z \rightarrow 1 / z$. Let $R=-p / q$, where $p, q$ are polynomials with no common factors.

2.2. Proposition. A point $\gamma$ in the finite plane is a critical point of (1.6) if and only if $\gamma$ is a pole of $R$. The point at infinity is a critical point of (1.6) if and only if $\operatorname{deg}(p) \geq 3+\operatorname{deg}(q)$.

Proof. Clearly, any critical point of (1.6) is a pole of $R$. Suppose $\gamma=0$ is a pole of $R$. A simple computation shows that if $f(0)=0$, then $f / f^{\prime}$ has a simple zero at 0 . Thus it is necessary to have $f(0) \neq 0$, and since $f^{\prime}=-f / R$, we have $f^{\prime}(0)=0$. Using $(2.1)$, the case for $\infty$ is obtained.

Note that $R$ can have a pole at $\infty$ without having a critical point there.

The degree of the rational function $N(\cdot, h)$ is $d=\max (\operatorname{deg}(p), 1+\operatorname{deg}(q))$. For $\zeta$ in $\widehat{\mathbf{C}}$, the equation $N(z, h)=\zeta$ has $d$ solutions. 
A solution $\varphi(t)$ which passes through a critical point (necessarily in finite time) is called a critical trajectory. See [2, §2]. At the critical points, smoothness and uniqueness break down.

\section{Dynamics on the Julia SETS}

For $\zeta$ in $\widehat{\mathbf{C}}$, the equation $N(z, h)=\zeta$ has $d$ solutions. We assume $d \geq 2$. Note in particular that if $\zeta=\infty$, then $z=\infty$ is a solution of multiplicity $d-\operatorname{deg}(q)$. If $z_{0}$ is given, let $z_{n}=z_{n}(h)$ denote a probabilistic sequence of backward iterates, where

$$
N\left(z_{n}, h\right)=z_{n-1}
$$

and $z_{n}$ is selected with probability $1 / d$. We assume the list of $d$ solutions of (3.1) has repetitions if there are multiple roots. For any $z_{0}$, let $N^{-1}\left(z_{0}, h\right)$ denote the set of preimages of $z_{0}$, those $z$ such that $N(z, h)=z_{0}$. Let $N^{-n}(h)$ denote the $n$-fold iteration of $N^{-1}(h)$. If $z_{0}$ is in $J(h)$, then $\bigcup_{1}^{\infty} N^{-n}\left(z_{0}, h\right)$ is dense in $J(h)$ [4, Corollary 4.7]. More specifically, if $\left\{z_{n}\right\}$ is a probabilistic sequence of backward iterates, starting at $z_{0}$ in $J(h)$, then $\left\{z_{n}\right\}$ is almost surely dense in $J(h)$. See $[1,8]$. The set of branches of $N^{-1}(h)$ along with the probabilities $1 / d$ is an iterated function system [1]. We shall call this method for computing points in $J(h)$ the IFS method.

We consider a single inverse step by studying solutions of

$$
N(z, h)=\zeta \text {. }
$$

We observe that, for $h$ sufficiently small, $z$ and $h R(z)$ cannot cancel terms, even if $\operatorname{deg}(p)=1+\operatorname{deg}(q)$, so that if $\zeta=\infty$ then the solutions of (3.2) consist of the poles of $R$ in the finite plane, and $z=\infty$. For $\varepsilon>0$ and $z_{0}$ in the finite plane, let $D\left(z_{0}, \varepsilon\right)=\left\{z:\left|z-z_{0}\right| z<\varepsilon\right\}$ and let $D(\infty, \varepsilon)=\{z:|z|>1 / \varepsilon\}$. Let $\gamma_{i}, i=1, \ldots, m$, denote the distinct poles of $R$. This set is not empty for $d \geq 2$, and consists of the zeros of $q$ (if any) and $\infty$ (if $\operatorname{deg}(p)>\operatorname{deg}(q)$ ). If $q$ has zeros let

$$
q(z)=\prod_{k=1}^{K}\left(z-s_{k}\right)^{n_{k}} .
$$

Thus the $s_{k}$ are a subset of the $\gamma_{i}$. Let

$$
P(\varepsilon)=\bigcup_{i=1}^{m} D\left(\gamma_{1}, \varepsilon\right), \quad P(\zeta, \varepsilon)=D(\zeta, \varepsilon) \cup P(\varepsilon) .
$$

3.3. Theorem. Let $\varepsilon>0$ be given. Then there exists $h_{0}(\varepsilon)>0$ such that for all $h,|h|<h_{0}(\varepsilon)$, and for all $\zeta$ in $\widehat{\mathbf{C}}$, all solutions of $(3.2)$ lie in $P(\zeta, \varepsilon)$.

Proof. Let $S(\varepsilon)=\widehat{\mathbf{C}}-P(\varepsilon)$. This is a compact subset of $\widehat{\mathbf{C}}$, and there exists $M=M(\varepsilon)>0$ such that $|R(z)| \leq M$ on $S(\varepsilon)$. If $|h|<\varepsilon / M$, then $|h R(z)|<\varepsilon$ on $S(\varepsilon)$. If also $|z-\zeta| \geq \varepsilon$, then

$$
|(z-\zeta)+h R(z)| \geq|z-\zeta|-|h R(z)|>0 .
$$

3.4. Corollary. Assume $|h|<h_{0}(\varepsilon) \varepsilon^{\operatorname{deg}(q)}$. Then every connected component of $P(\zeta, \varepsilon)$ in the finite plane contains as many solutions of (3.1) as zeros of $(-\zeta) q(z)$ 
Proof. Rewrite (3.2) as

$$
(z-\zeta) q(z)+h p(z)=0 .
$$

On the boundary of $P(\zeta, \varepsilon)$, we have $|(z-\zeta) q(z)| \geq \varepsilon^{1+\operatorname{deg}(q)}$. With the above condition on $|h|$, the conditions of Rouche's theorem are satisfied on the boundary of each connected component: $|(z-\zeta) q(z)|>|h p(z)|$.

The following is a simple consequence of these results.

3.5. Theorem. If $\zeta$ is in the finite plane and $D(\zeta, \varepsilon)$ is disjoint from $D\left(\gamma_{k}, \varepsilon\right)$, then for $h$ sufficiently small the solution $z$ of (3.2) has probability $n_{k} / d$ of being in $D\left(\gamma_{k}, \varepsilon\right)$ and probability $1 / d$ of being in $D(\zeta, \varepsilon)$. If $\infty$ is a pole of $R$, it has probability $[d-1-\operatorname{deg}(q)] / d$ of being in $D(\infty, \varepsilon)$.

Thus, for $h$ small, the backward iterates of $N(\cdot, h)$ have a probability distribution on $J(h)$, which is close to an atomic measure concentrated on the poles of $R$. Of particular interest is the case in which $\operatorname{deg}(p)=1+\operatorname{deg}(q)$. This then applies to Newton's method for polynomials. In this case, $\infty$ is a pole of $R$, but all $d$ solutions of (3.2) for $\zeta$ in the finite plane are accounted for in the finite plane, so even if $\infty$ is in $J(h)$, the sequence of backward iterates has small probability of reaching a neighborhood of $\infty$.

Since all poles of $R$ in the finite plane are critical points of the differential equation (1.1), we see that the part of $J(h)$ in the finite plane becomes concentrated, in the sense of the invariant measure, around those points where uniqueness and smoothness of solutions fail to hold.

\section{Distribution AlONg CRITICAL tRAJECTORIES}

In the special case that

$$
f(z)=\prod_{j=1}^{J}\left(z-\rho_{j}\right)^{A_{j}},
$$

where $\rho_{j}, j=1, \ldots, J$, are points in the finite plane and $A_{j}, j=1, \ldots, J$, are complex numbers with $\operatorname{Re} A_{j}>0$, we show that there is further structure to the distribution of $J(h)$ around the critical points. We show that for small $h, J(h)$ is confined to narrow strips containing those parts of the critical trajectories which will reach critical points in forward time. This is based on a study of Euler's method for (1.6). In general, Euler's method is local; a solution can be approximated for only a short time interval. See [6, Theorem 1.1, p. 3]. We show that for (1.6), if $B$ denotes a set of initial points $\eta$ and if $T>0$ is arbitrary, such that the set $\{\varphi(t, \eta)\}$ for $\eta$ in $B$ and $0 \leq t \leq T$ is bounded away from the poles of $R$, then for any tolerance $\delta>0$, Euler's method starting at $\eta$ will approximate $\varphi(t, \eta)$ to this tolerance for $0 \leq t \leq T$, provided $h$ is sufficiently small, and this holds uniformly in all such $\eta$. There is numerical evidence that the main result of this section holds under more general conditions.

Assuming (4.1), it is easily verified that

$$
R(z)=-\frac{\prod_{j=1}^{J}\left(z-\rho_{j}\right)}{\sum_{j=1}^{J} A_{j} \prod_{k \neq j}\left(z-\rho_{k}\right)},
$$


and in a neighborhood of each zero $\rho_{1}$,

$$
R(z)=-\left(z-\rho_{1}\right) g_{1}(z) / A_{1},
$$

where $g_{1}\left(\rho_{1}\right)=1$. Since $\operatorname{Re} A_{j}>0$, we see that $\operatorname{deg}(p)=1+\operatorname{deg}(q)$, so that $\infty$ is a pole of $R$ but not a critical point of (1.6).

4.4. Proposition. Assume (4.1). For the continuous system (1.6), each $\rho_{j}$ is an asymptotically stable equilibrium solution and $\infty$ is a repelling equilibrium solution. For the discrete system (1.7), for $h$ positive and sufficiently small, each $\rho_{j}$ is an attracting fixed point and $\infty$ is a repelling fixed point.

Proof. From (4.3) we see that $R^{\prime}\left(\rho_{1}\right)=-1 / A_{1}$, which lies in the left halfplane. Thus by standard linearization theory, $\rho_{1}$ is asymptotically stable. Since $N^{\prime}\left(\widetilde{z}_{1}\right)=\left(A_{1}-h\right) / A_{1}$, we see that $\rho_{1}$ is attracting if $A_{1}$ is closer to $h$ than to 0 . In particular, this holds for $0<h<2 \operatorname{Re} A_{1}$. As for the point at infinity, let $A=A_{1}+\cdots+A_{J}$. Then

$$
R(z)=\frac{-z[1+g(z)]}{A}, \quad N(z)=z\left\{1-\frac{h[1+g(z)]}{A}\right\},
$$

where $g \rightarrow 0$ uniformly as $z \rightarrow \infty$. Thus the derivative at 0 of $-z^{2} R(1 / z)$ lies in the right half plane, so $\infty$ is a repelling equilibrium solution for (1.6), and a repelling fixed point for (1.7), provided $0<h<2 \operatorname{Re} A$.

We now show that there is a basin of attraction for the set of attracting fixed points of $N(\cdot, h)$ which is independent of $h$ for $h$ sufficiently small.

4.5. Theorem. There exist $\rho>0$ and $h_{0}>0$ such that for all $h, 0<h<h_{0}$, if $\left|z-\rho_{j}\right|<\rho$, then the iterates of $N(z, h)$ converge to $\rho_{j}$.

Proof. Assume $z=0$ is a fixed point of $N(\cdot, h)$. Then $f(z)=z^{a} g(z)$ where $\operatorname{Re} a>0$ and $g$ is analytic at $0, g(0) \neq 0$. Let $\psi=g^{\prime} / g$. Then

$$
N(z, h)=z \frac{a+z \psi(z)-h}{a+z \psi(z)},
$$

so $|N(z, h) / z|<1$, provided $0<h<2 \operatorname{Re}[a+z \psi(z)]$. Select $\rho$ so that $|z \psi(z)|<\operatorname{Re} a / 2$ for $|z|<\rho$. Then $|N(z, h) / z|<1$ for all such $z$. Since $N$ has only finitely many fixed point, the smallest $\rho$ for these fixed points is still positive, and $h_{0}$ can be chosen as the smallest of $\operatorname{Re} A_{j}$.

We consider the approximation of a single solution.

4.6. Proposition. Let $T, \Delta, \delta$ be given, all positive, and $\delta<\Delta$. Assume $\varphi(t, \eta)$ satisfies the condition that if $|z-\varphi(t, \eta)|<2 \Delta$ for any $t, 0 \leq t \leq T$, then for some $M=M(T, \Delta)$, we have $|R(z)| \leq M,\left|R^{\prime}(z)\right| \leq M$. Then there exist $\varepsilon_{1}(T, \Delta, \delta)>0, h_{1}(T, \Delta, \delta)>0$ such that if $\left|z_{0}-\eta\right|<\varepsilon_{1}$ and $0<h<h_{1}$, then $\left|z_{k}(h)-\varphi(k h, \eta)\right|<\delta$ for all $k$ such that $k h \leq T$.

Proof. Let $\left|z_{0}-\eta\right|=\varepsilon<\Delta$, and let $z_{k}(h)$ be given by (1.2). By induction, as long as $\left|z_{n}-\eta\right|<2 \Delta$, we have $\left|z_{n}-\eta\right|<\varepsilon+n h M$. Thus $n h \leq(2 \Delta-\varepsilon) / M$. Let $T_{1}=T_{1}(\Delta)=\Delta / M$. Then $\left|z_{n}-\eta\right| \leq 2 \Delta$ for $n h \leq T_{1}$. Using Taylor's theorem for vector-valued functions, we have

$$
\varphi((k+1) h, \eta)=\varphi(k h, \eta)+h R(\varphi(k h, \eta))+\frac{h^{2} E_{k}}{2}
$$


where $\left|E_{k}\right| \leq 2 M^{2}$. Using Taylor's theorem for analytic functions, we have

$$
\left|R\left(z_{k}\right)-R(\varphi(k h, \eta))\right| \leq M\left|z_{k}-\varphi(k h, \eta)\right| .
$$

Let $e_{k}=z_{k}-\varphi(k h, \eta)$. Then as in [9, p. 27], we have

$$
\left|e_{n}\right| \leq \varepsilon e^{T_{1} M}+h M\left(e^{T_{1} M}-1\right)
$$

for $n h \leq T_{1}$. By selecting $\varepsilon, h$ sufficiently small, we have $\left|e_{k}\right|<\delta$ for $k h \leq$ $T_{1}$. Let $\nu$ be that integer such that $\nu T_{1} \leq T<(\nu+1) T_{1}$. Then the above process can be carried out $\nu$ times for $\varepsilon<\varepsilon_{1}(T, \Delta, \delta), 0<h<h_{1}(T, \Delta, \delta)$.

Note that uniformly bounding $|R|,\left|R^{\prime}\right|$ is equivalent to keeping $z$ uniformly bounded away from the poles of $R$. Also, since $T_{1}, \varepsilon_{1}$ depend only on $\Delta, \delta$, and $M$, we see that this result holds uniformly over sets of trajectories.

4.7. Theorem. Let $K$ denote a set in the finite plane such that, for all $\eta$ in $K$, all trajectories $\varphi(t, \eta)$ are uniformly bounded away from the poles of $R$ for $0 \leq t \leq T$. Then for $\delta>0$ but sufficiently small, the Euler approximations $z_{k}(h)$ satisfy $\left|z_{k}(h)-\varphi(k h, \eta)\right|<\delta$ for all $k$ such that $k h \leq T$.

4.8. Theorem. Let $K$ be a compact set such that, for each $\eta$ in $K$, the solution $\varphi(t, \eta)$ is free of critical points for $t \geq 0$. Let $\rho$ be as in Theorem 4.5. Then there exists $T=T(K)$ such that $\left|\varphi(T, \eta)-\rho_{j}\right| \leq \rho / 2$ for all $u_{0}$ in $K$ and some equilibrium solution $\rho_{j}$.

Proof. Since $\operatorname{Re} A_{j}>0$ for all $j$, there are no periodic solutions of (1.6) and there are no limit cycles [2, Theorem 2.16]. Thus each solution of (1.6) tends to one of the equilibrium solutions in forward time. Let

$$
T(\eta, \rho)=\inf \left\{t \geq 0:\left|\varphi(t, \eta)-\widetilde{z}_{j}\right| \leq \rho / 2 \text {, some } j\right\} .
$$

Then $T(\eta, \rho)<\infty$. Since $K$ is closed, there is a $\delta>0$ such that, for any critical point $\gamma$ of $f,|\varphi(t, \eta)-\gamma| \geq 2 \delta$ for $t \geq 0$. (If $\varphi\left(t_{n}, \eta_{n}\right) \rightarrow \gamma$, then $\eta_{n}$ [or a subsequence] converges to a point $\tilde{\eta}$ in $K$. Then $\varphi\left(t_{n}, \tilde{\eta}\right) \rightarrow \gamma$, contradicting the definition of $K$.) Thus for $\eta$ in $K$ all solutions are continuously dependent on initial data, so $T(\eta, \rho)$ is continuous on $K$. Thus $T(K)=\sup \{T(\eta, \rho)\}$ is finite.

4.9. Theorem. Let $K$ be a compact set such that, for all $\eta$ in $K$ and all $t \geq 0$, the trajectories $\varphi(t, \eta)$ are a distance $\delta>0$ from the critical points of (1.6). Then there exists $h_{0}=h_{0}(K)$ such that $K$ is free of points of $J(h)$ for $0<h<h_{0}$.

Proof. By Theorem 4.8, there exists $T>0$ such that $\varphi(T, \eta)$ is in an attracting neighborhood of some $\rho_{j}$, uniformly over $\eta$ in $K$. By Theorem 4.7, for $h$ sufficiently small the iterates of $N(\cdot, \eta)$ are eventually in an attracting neighborhood of some $\rho_{j}$, so the sequence of iterates is normal at each $\eta$ in $K$. Thus no point of $K$ can be in $J(h)$ for $h$ sufficiently small.

Thus, to find points of $J(h)$, it is necessary to look at neighborhoods of points on critical trajectories which have yet to pass through critical points in forward time.

\section{EXAMPL:i}

Let

$$
f(z)=\frac{1}{3} z^{3}-\frac{3}{2} z^{2}+2 z
$$




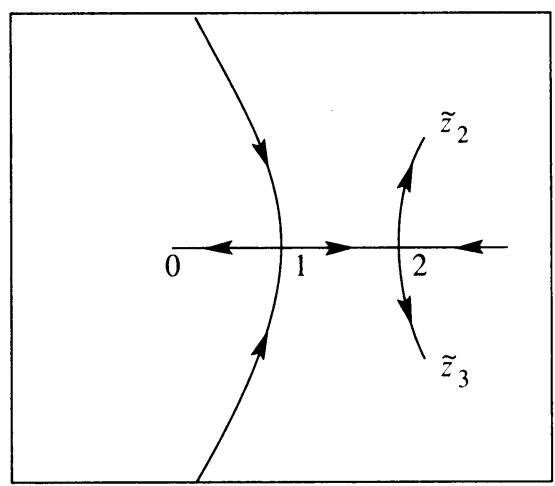

FigURe 1

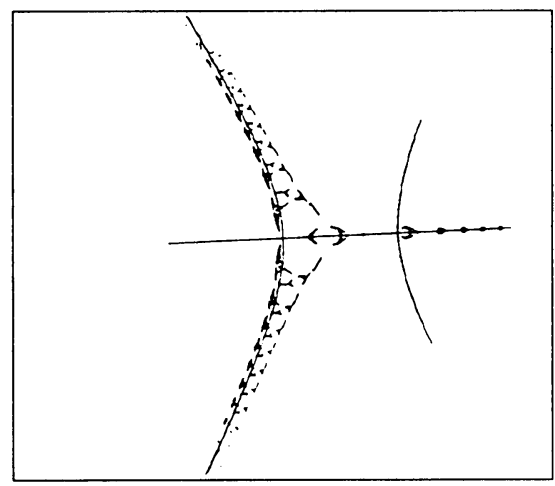

FIGURE 3. $h=0.2$

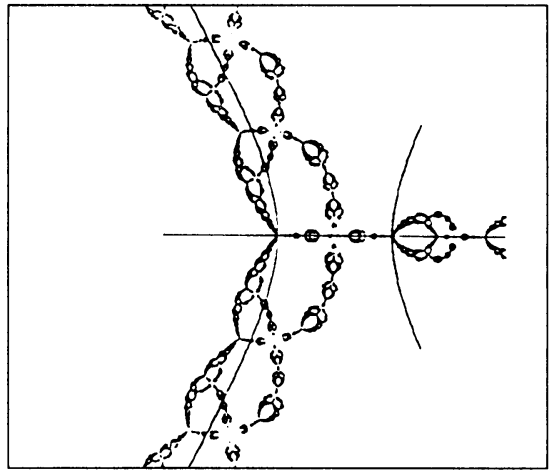

FIGURE 2. $h=1$

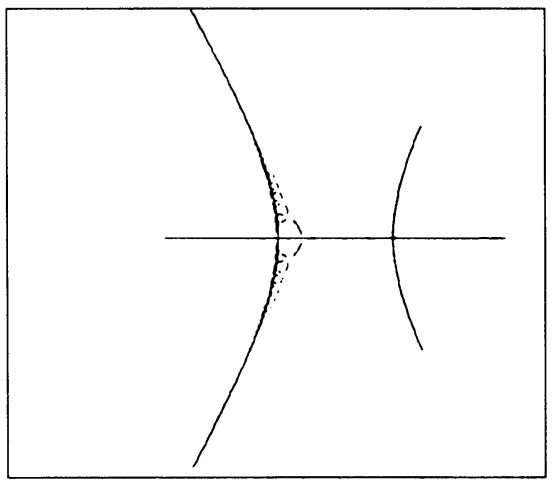

FIGURE 4. $h=0.5$

Then $f$ has zeros at $\rho_{1}=0$, at

$$
\rho_{2}=\frac{9}{4}+\frac{i \sqrt{15}}{4}
$$

and at $\rho_{3}$ which is the conjugate of $\rho_{2}$. The critical points are at $\gamma_{1}=1$, $\gamma_{2}=2$. The critical trajectories and their orientations in forward time are shown in Figure 1. Figures 2, 3, and 4 show $J(h)$ for $h=1, h=0.2$, and $h=0.05$, respectively. The critical trajectories were drawn using a noniterative method described in $[2, \S 6]$, and the Julia sets were plotted using the IFS method. The plots do not show regions of $J(h)$ which have low probability according to the invariant measure.

\section{REFERENCES}

1. M. F. Barnsley and S. Demko, Iterated function systems and the global construction of fractals, Proc. Roy. Soc. London Ser. A 399 (1985), 243-275.

2. H. E. Benzinger, Plane autonomous systems with rational vector fields, to appear, Trans. Amer. Math. Soc. 326 (1991), 465-484.

3. H. E. Benzinger, S. A. Burns, and J. I. Palmore, Chaotic complex dynamics and Newton's method, Phys. Lett. A 119 (1987), 441-446.

4. P. Blanchard, Complex analytic dynamics on the Riemann sphere, Bull. Amer. Math. Soc. (N.S.) 11 (1984), 85-141. 
5. H. Brolin, Invariant sets under iteration of rational functions, Ark. Mat. 6 (1965), 103-144.

6. E. A. Coddington and N. Levinson, Theory of ordinary differential equations, McGraw-Hill, New York, 1955.

7. J. Curry, L. Garnett, and D. Sullivan, On the iteration of a rational function, computer experiments with Newton's method, Comm. Math. Phys. 91 (1983), 267-277.

8. J. H. Elton, An ergodic theorem for iterated maps, Ergodic Theory and Dynamical Systems 7 (1987), 481-488.

9. P. Henrici, Discrete variable methods in ordinary differential equations, Wiley, New York, 1962.

10. H. Th. Jongen, P. Jonker, and F. Twilt, The continuous, desingularized Newton method for meromorphic functions, Acta. Appl. Math. 13 (1988), 81-121.

11. H.-O. Peitgen and P. H. Richter, The beauty of fractals, Springer-Verlag, Berlin, 1986.

Department of Mathematics, University of Illinois, Urbana, ILlinoIs 61801 